\title{
A prospective examination of glycaemic control in patients with insulin requiring diabetes receiving intermittent and continuous enteral feeding in an acute hospital setting
}

\author{
P. M. Murphy ${ }^{1}$, E. Moore ${ }^{2}$ and D. Flanagan ${ }^{2}$ \\ ${ }^{1}$ Department of Nutrition and Dietetics Plymouth Hospitals NHS Trust, Plymouth PL6 8DH, UK and ${ }^{2}$ Department of \\ Diabetes and Endocrinology, Plymouth Hospitals NHS Trust, Plymouth PL6 8DH, UK
}

We recently found a high incidence of hypoglycaemia in diabetes patients receiving subcutaneous insulin and enteral feeding over a 20 -h period. The aim of the present study was to examine the effect of intermittent and continuous enteral feeding on glycaemic control.

Forty patients with insulin requiring diabetes were referred for enteral tube feeding over a 22-month period, 28 were evaluable. Only patients with normal gastrointestinal function and full nutritional needs met by enteral tube feeding were included. Thirteen consecutive patients were prescribed a basal bolus insulin regimen with intermittent daytime enteral tube feeding. A further 15 consecutive patients received continuous feeding and long acting insulin. Blood glucose was measured prior to commencing feed, midway though each feeding episode and overnight for intermittently fed patients. During continuous feeding, blood glucose monitoring was recommended four times daily. All patients were fed a standard $1 \mathrm{kcal} / \mathrm{ml}$ formula to meet nutritional requirements. Patients were reviewed daily during enteral feeding by a dietitian or diabetes specialist nurse. Descriptive statistics, chi-squared analysis and analysis of variance compared data between groups.

\begin{tabular}{lcccr}
\hline & Number of observations & Blood glucose $<4 \mathrm{mmol} / \mathrm{l}$ & Blood glucose 4-12 mmol/l & Blood glucose $>12 \mathrm{mmol} / \mathrm{l}$ \\
\hline 20-h feed & 135 & $10(7 \%)$ & $58(43 \%)$ & $67(50 \%)$ \\
Intermittent feed & 817 & $33(4 \%)$ & $486(59 \%)$ & $298(36 \%)$ \\
Continuous feed & 702 & $24(3 \%)$ & $347(49 \%)$ & $331(47 \%)$ \\
\hline
\end{tabular}

Hospital mortality was $40 \%$ overall (16 of 40). Mean duration of enteral feeding was 15 and 11 days for intermittent and continuous feed groups, respectively. Mean length of hospital stay was 45 days. No difference in the incidence of hypo-, hyper- or normoglycaemia was found between intermittent and continuous feed groups. Feed interruption was the cause of $12 \%$ and $29 \%$ recorded episodes of hypoglycaemia in the intermittent and continuous feed groups, respectively.

Continuous enteral feeding does not eliminate hypoglycaemia in clinical practice. Strategies to prevent dislodgement of feeding tubes and the availability of appropriate protocols for use when feed is interrupted may be beneficial. Intensive monitoring of blood glucose and review by a multidisciplinary team is essential to detect and treat hypo- and hyperglycaemia. 